Reprod. Nutr. Dévelop., 1985, 25 (3), 555-566.

\title{
Dégénérescence cellulaire intestinale chez I'Annélide Polychète Perinereis cultrifera au cours de l'épitoquie et après ablation du cerveau
}

\author{
A. DAKHAMA ( $\left.{ }^{1}\right)$, A. DHAINAUT (2) \\ avec la collaboration technique de Françoise BONET
}

Laboratoire de Biologie Animale, L.A. C.N.R.S. No 148, Endocrinologie des Invertébrés. Université de Lille I, 59655 Villeneuve-d'Ascq Cedex, France.

Summary. Cellular degeneration in the gut of Annelida polychaeta Perinereis cultrifera during epitoky and after brain removal.

Near epitoky (breeding stage), the gut epithelium showed the characteristic features of a degenerative process (appearance of cytolysomes and autophagic vacuoles) and a few degraded cells were shed into the gut lumen. Many organelles became rich in acid phosphatases.

Brain removal triggered similar cytological changes. A drop in cell renewal was revealed by autoradiography following $3-\mathrm{H}$-thymidine incorporation, and biochemical data showed an increase in the phosphatase level and a decline of DNA synthesis. This programmed cell death seemed to be related to a drop in brain endocrine activity.

\section{Introduction.}

Les Néréidiens (Annélides Polychètes) sont des animaux monothéliques. A l'approche de la période de reproduction, ils présentent d'importantes modifications morphologiques (augmentation de la taille des yeux, élargissement des parapodes, acquisition de soies en palettes) liées à une adaptation à la vie pélagique. Ces transformations somatiques désignées sous le nom d'épitoquie sont contrôlées, de même que l'évolution des cellules germinales, par une hormone d'origine cérébrale (Durchon, 1952). L'ablation du cerveau déclenche en effet le processus d'épitoquie (Durchon, 1948, 1952) et dans les conditions naturelles, il a été démontré que l'activité endocrine du cerveau diminue progressivement à l'approche de la maturation (Porchet, 1972).

Parallèlement aux transformations précédemment décrites, il a été signalé (revue in Clark, 1961) que lors de la reproduction, le tube digestif présente une

(1) Adresse présente : Université Cadi Ayyad, Faculté des Sciences, Marrakech (Maroc).

(2) Demandes de tirés à part à adresser à A. DHAINAUT. 
dégénérescence de l'épithélium intestinal, qui s'accompagne d'un arrêt de l'alimentation. Par son déclenchement à un stade bien particulier de la vie de l'animal cette dégénérescence semble résulter d'un processus de mort cellulaire programmée selon le concept de Lockshin et Williams (1964). Il a été bien établi chez les Amphibiens (Hourdry et Dauca, 1977) et chez les Insectes (Beaulaton et Lockshin, 1982 ; Andriès, 1983) que ce phénomène est conditionné par un facteur hormonal. Il était intéressant de rechercher si chez les Annélides ce processus était lié aux modifications endocrines résultant de l'ablation du cerveau. Dans cette optique, l'influence du jeûne devait également être examinée.

La structure de l'épithélium intestinal des Nereis atoques (ou immatures) a fait l'objet récemment de plusieurs études (Punin et Lukyanova, 1984 a et b). Le présent travail a étudié les modifications structurales et métaboliques survenant au cours de l'épitoquie naturelle ou provoquée par l'ablation du cerveau. L'évolution du renouvellement cellulaire a été appréciée par incorporation de thymidine tritiée suivie d'une étude autoradiographique et du dosage des acides nucléiques.

\section{Matériel et méthodes.}

Perinereis cultrifera (Grübe) est une Annélide Polychète vivant dans les rochers de la zone intertidale. La récolte a été effectuée à Luc-sur-Mer (Calvados) en période de grande marée. Au laboratoire, les vers sont isolés dans des récipients contenant de l'eau de mer renouvelée chaque jour. Ils sont maintenus à une température d'environ $13^{\circ} \mathrm{C}$.

Expérimentation. - Après anesthésie au MS 222 (Laboratoire Sandoz) à la concentration de $5 \mathrm{~g} / \mathrm{l}$ d'eau de mer, l'ablation des prostomiums est réalisée, sous loupe binoculaire, à l'aide de ciseaux de Pascheff.

Microscopie électronique. - Après prélèvement, les petits fragments d'intestin sont fixés pendant $3 \mathrm{~h}$ à $4{ }^{\circ} \mathrm{C}$ dans une solution de glutaraldéhyde à $3 \%$ dans du tampon phosphate additionné de $2,5 \mathrm{M}$ de $\mathrm{NaCl}$. Après lavage dans la même solution tampon, les pièces sont post-fixées au tétroxyde d'osmium pendant $1 \mathrm{~h}$ à $4^{\circ} \mathrm{C}$. Après déshydratation par l'acétone, les pièces sont enrobées dans l'araldite et coupées au microtome Porter-Blum ou LKB.

L'activité de la phosphatase acide a été recherchée selon la technique de Miller et Palade (1964) modifiée pour le matériel marin par Bertout (1976).

Autoradiographie. - Les animaux destinés à l'étude des synthèses d'ADN ont reçu une dose de $50 \mu \mathrm{Ci} / g$ de thymidine ${ }^{3} \mathrm{H}(25 \mathrm{Ci} / \mathrm{mM}$ - CEA). Après un temps d'incorporation de $24 \mathrm{~h}$, les fixations sont réalisées au Carnoy. Les coupes sont recouvertes d'émulsion llford $\mathrm{K} 5$.

\section{Dosages biochimiques}

Synthèses d'ADN : L'injection de thymidine ${ }^{3} \mathrm{H}(20 \mu \mathrm{Ci} / \mathrm{g})$ est effectuée $24 \mathrm{~h}$ avant l'isolement de l'intestin. La radioactivité est déterminée au niveau de l'hydrolysat d'ADN. Le dosage de celui-ci est réalisé par la méthode de Giles et Myers (1965). Les résultats sont exprimés en $\mathrm{cpm} / \mu \mathrm{g} \mathrm{d}^{\prime} \mathrm{ADN}$. 
Phosphatase acide: Selon la technique de Wattiaux et De Duve (1956), $0,1 \mathrm{ml}$ de fractions cellulaires est incubé pendant $30 \mathrm{mn}$ à $37^{\circ} \mathrm{C}$ en présence de $0,9 \mathrm{ml}$ de $\beta$-glycérophosphate de $\mathrm{Na} 50 \mathrm{mM}$ dans le tampon acétate de sodium $50 \mathrm{mM}$ contenant $0,07 \%$ de Triton $X-100$. La réaction est arrêtée par addition de $1 \mathrm{ml}$ de TCA $10 \%$. Les tubes sont centrifugés à basse vitesse et la quantité de phosphate inorganique est dosée dans le surnageant. L'activité enzymatique est exprimée en $\mu$ moles de phosphate inorganique libérées par minute et par $\mathrm{mg}$ de protéines.

\section{Résultats.}

\section{1) Evolution cytologique des cellules de l'épithélium intestinal.}

L'épithélium intestinal comporte différentes catégories cellulaires : les entérocytes, les cellules glandulaires muqueuses, les cellules ciliées, les fibres nerveuses. Seule l'évolution de la structure des entérocytes sera envisagée dans cette étude.

Structure normale et influence du jeûne. - Chez les animaux jeunes, les entérocytes s'étendent sur toute la hauteur de l'épithélium intestinal et se caractérisent par la présence de microvillosités bien développées. Le cytoplasme apical renferme de nombreuses mitochondries ainsi que des organites apicaux denses, de forme allongée, orientés perpendiculairement à la membrane plasmique (fig. 1). Dans la zone sous-jacente, sont localisés des corps multivésiculaires et des vacuoles qui paraissent liés au processus de digestion intracellulaire (Dakhama et Dhainaut, 1985). Ces organites, en nombre très peu élevé (3 à 4 par section de cellule) ont une taille ne dépassant pas un diamètre de 0,6 $\mu \mathrm{m}$. De la phosphatase acide est détectée à leur niveau, les produits de réaction étant généralement localisés contre la paroi des vacuoles (fig. 2). Les lobules lipidiques occupent la zone basale des entérocytes (fig. 3). De rares rosettes de glycogène sont disséminées dans le cytoplasme.

L'intestin des animaux soumis à un jeûne de 20 jours à 1 mois ne présente pas de modifications notables par rapport aux témoins. Il faut attendre un délai de 4 à 5 mois pour observer une diminution de la hauteur de l'épithélium et une légère réduction du nombre des microvillosités. Toutefois, les organites apicaux denses persistent (fig. 4). A ce stade apparaissent des cytolysomes dont le contenu renferme des granules lipidiques et des formations pseudomyéliniques. Des vacuoles apparaissent dans le cytoplasme, le plus souvent elles renferment un matériel d'aspect homogène; dans certains cas une auréole corțicale dense caractérise le contenu de ces formations (fig. 4).

Epitoquie naturelle. - Le degré d'évolution de l'épitoquie peut être apprécié par les modifications morphologiques des parapodes (développement des lamelles, apparition des soies en palettes).

En début d'épitoquie, les modifications initiales des entérocytes sont essentiellement observées dans la zone apicale. Les microvillosités perdent leur structure régulièrement cylindrique et leur forme devient irrégulière. Les organites apicaux denses tendent à s'allonger puis à se désorganiser; leur nombre régresse 


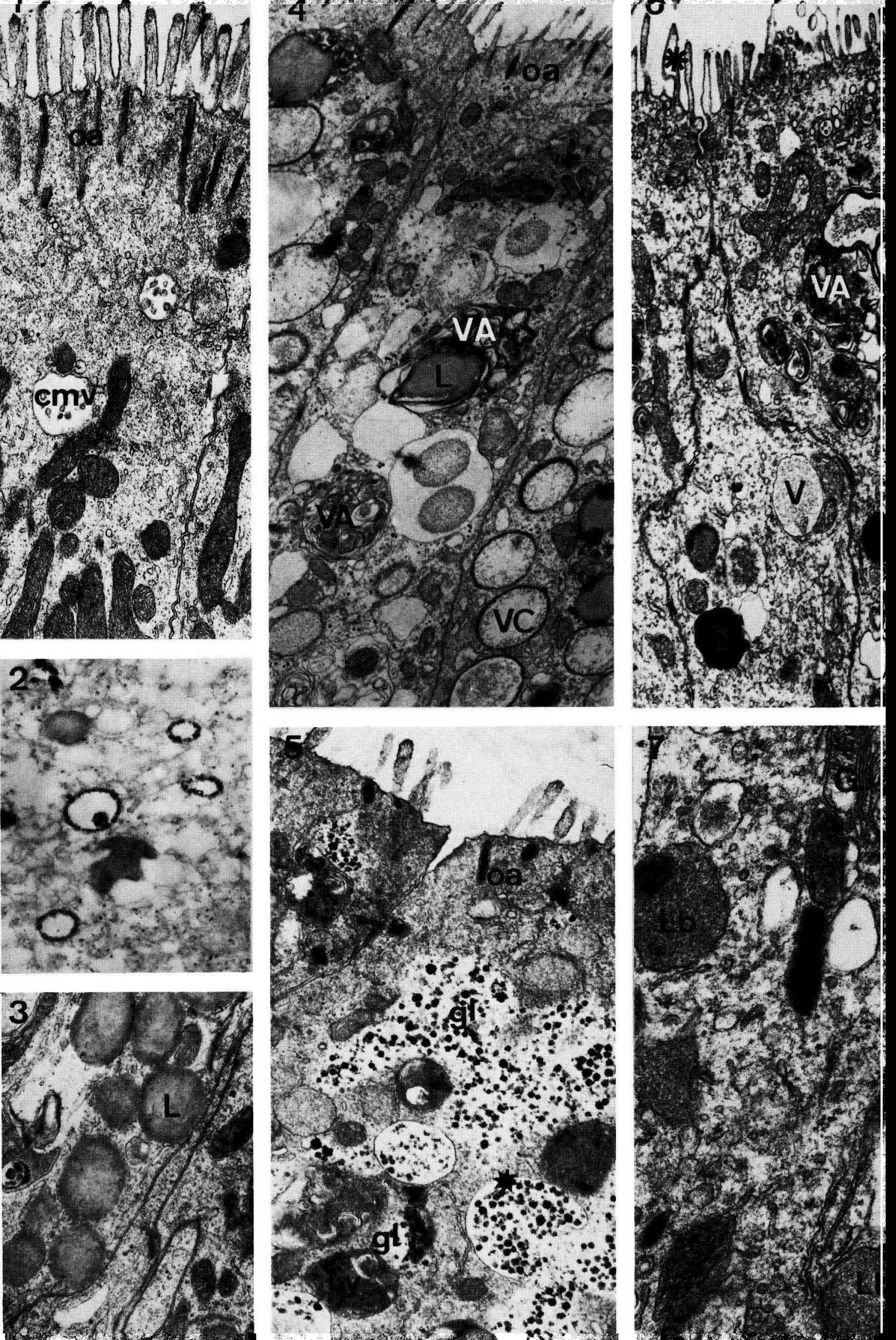


considérablement (fig. 5). Dans le cytoplasme sous-jacent, on note une profilération de rosettes de glycogène, le plus souvent réparties en plages d'étendue variée, mais parfois englobées à l'intérieur de systèmes membranaires (fig. 5). De nombreux organites à structure lysosomiale font leur apparition. Enfin, vers la zone basale de la cellule, on note une tendance à la fusion des lobules lipidiques en des éléments de grande taille.

A un stade plus avancé, la morphologie des dictyosomes se modifie. Les saccules golgiens présentent un aspect dilaté à leur extrémité. Leur contenu renferme un matériel dense de structure fibrillaire (fig. 7 et 8). Des corps de densité très élevée sont fréquemment observés à proximité des dictyosomes dont ils dérivent vraisemblablement (fig. 7). Ces éléments semblent s'intégrer dans des organites à matrice homogène renfermant des noyaux excentrés très denses. Des vacuoles autophagiques apparaissent. De grande taille (2 à $3 \mu \mathrm{m}$ ), leur formation résulte de la séquestration sous une enveloppe plus ou moins complexe d'une portion du cytoplasme. Leur contenu est surtout riche en systemes membranaires, des mitochondries profondément dégradées peuvent parfois y être décelées.

A maturité complète, la plupart des entérocytes conservent une morphologie comparable à celle décrite précédemment (fig. 8 et 11). Toutefois certaines cellules de l'épithélium sont affectées par une dégénérescence plus poussée (fig. 11). Ces cellules font hernie dans la lumière intestinale. Elles ont complètement perdu leurs microvillosités. Leur noyau est en picnose et leur cytoplasme est caractérisé à la fois par la présence de nombreuses vacuoles autophagiques et par une importante vacuolisation des citernes de réticulum. Des rejets de ces cellules dans la lumière intestinale ont été observés en microscopie photonique mais l'importance de ce processus semble toutefois limitée.

Epitoquie expérimentale. - Après 20 jours d'évolution, les modifications de l'épithélium sont comparables à celles observées chez les vers parvenus, dans les conditions naturelles, en début d'épitoquie. Au pôle apical des entérocytes les

FIG. 1. - Détail de la région apicale d'un entérocyte de ver immature. Les microvillosités sont bien développées et des organites à contenu dense (oa) sont localisés près de la membrane plasmique. Quelques corps multivésiculaires $(\mathrm{cmv})$ sont présents. $\times 20000$.

FIG. 2. - Mise en évidence de phosphatase acide dans les vacuoles apicales d'un ver immature. $\times 8000$.

FIG. 3. - Lobules lipidiques de la zone basale d'entérocytes de ver immature (L). $\times 10000$.

FIG. 4. - Ver soumis à l'influence du jeône pendant 5 mois. Les organites denses (oa) persistent. On constate l'apparition de vacuoles à auréole corticale dense (VC) et de vacuoles autophagiques (VA) renfermant des lipides (L.) X 15000.

FIG. 5. - Début de l'épitoquie naturelle. Raréfaction des organites apicaux (oa). Apparition de structures Iytiques (Ly). Noter l'abondance de rosettes de glycogène parfois entourées d'une membrane (astérisque). $\times 24000$.

FIG. 6. - Epitoquie naturelle (stade sub mature). La dégénérescence épithéliale se manifeste par l'apparition d'anomalies dans la structure des microvillosités (astérisque), la disparition des organites corticaux, la présence dans le cytoplasme de vacuoles à contenu hétérogène (V) et de vacuoles autophagiques (VA). X 12000 .

FIG. 7. - Epitoquie naturelle (stade sub mature). Elargissement des extrémités des saccules golgiens (G) qui renferment un matériel dense de structure fibrillaire. Noter l'existence de lobules (Lb) à noyau dense. $\times 35000$. 


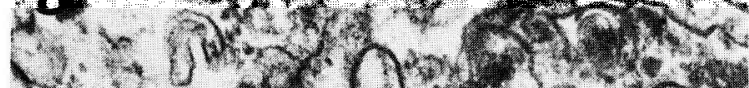
$M-14060 \%$

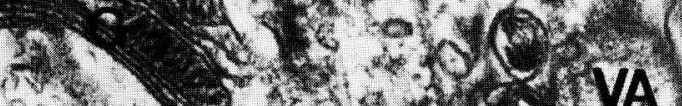
vo ona 10. o s.t.

is

SC. $x)$ L Ly.
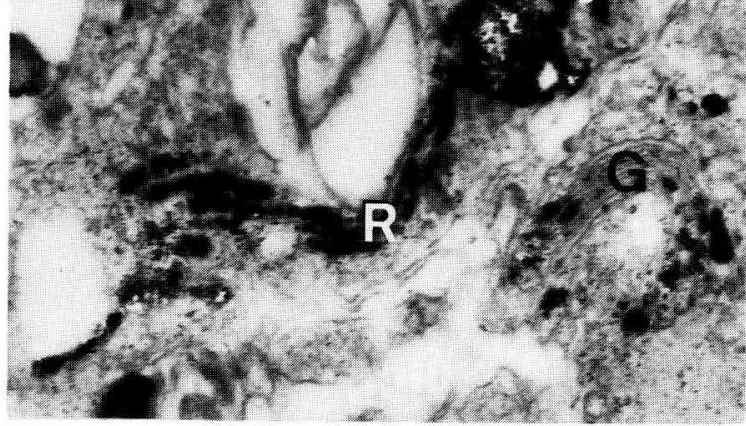

$3 x^{2}$

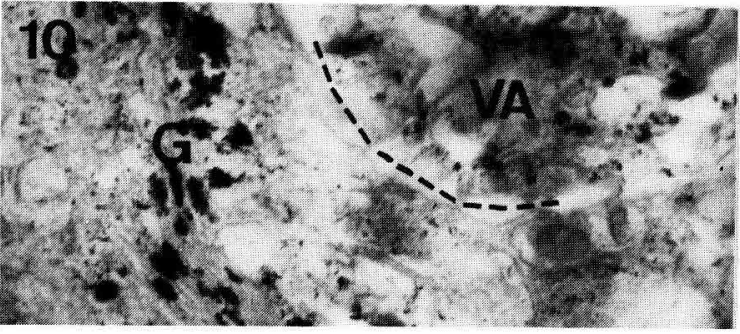

11

8.

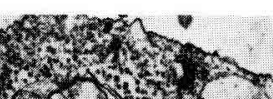

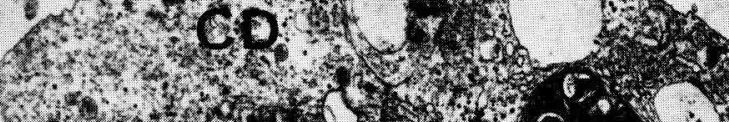

(1)

20 1,

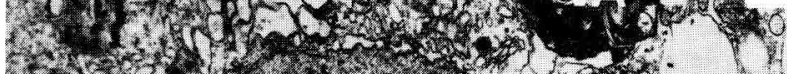

$x_{0}-2,3$

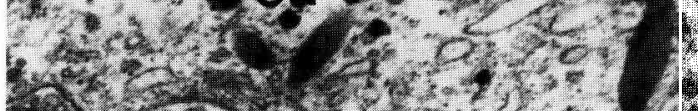
2.1.5.

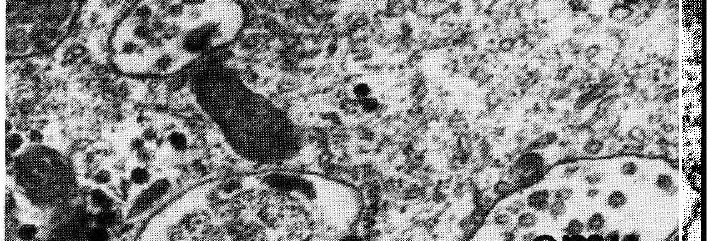

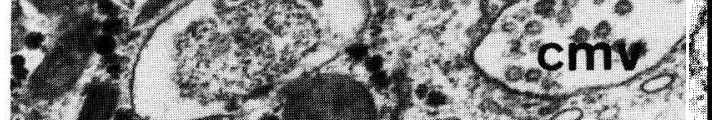

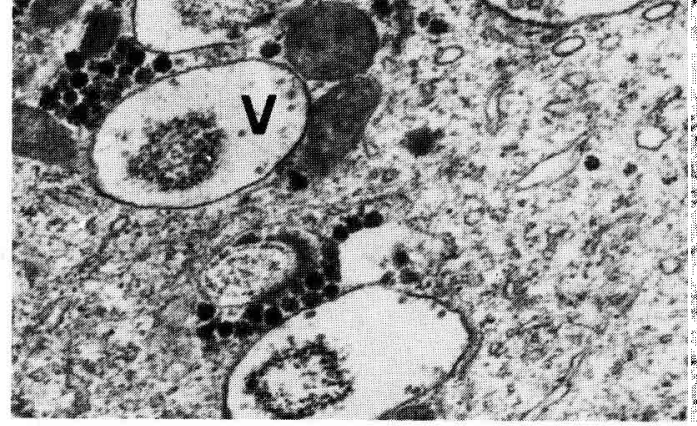

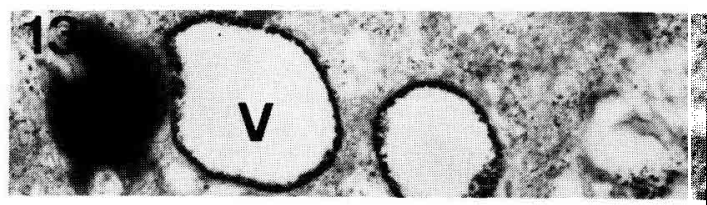

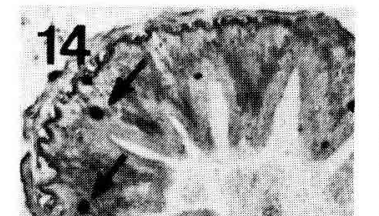

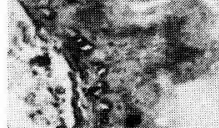

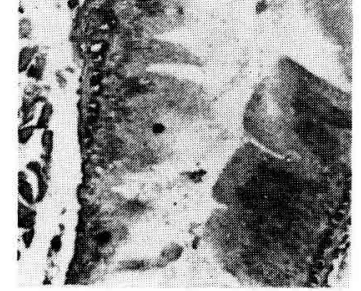

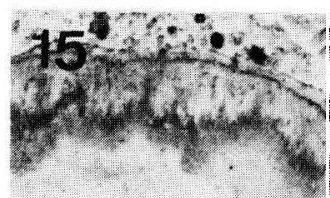

Xe

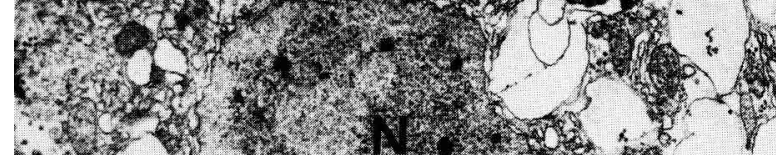
3

tions?

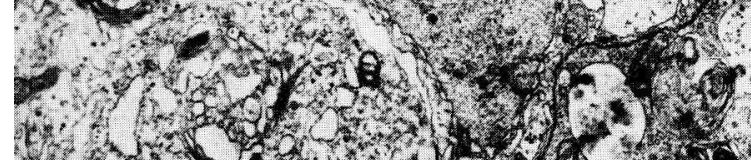

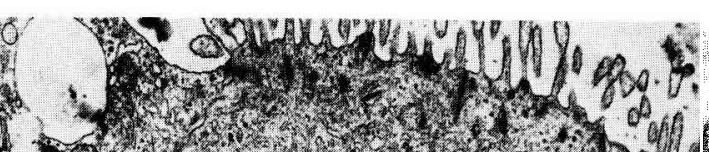
(1) 3. (4) (x) 1

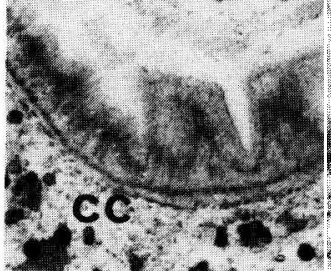


microvillosités acquièrent une structure irrégulière et leur nombre tend à se réduire. Les formations apicales denses sont modifiées dans leur organisation et régressent (fig. 12). Le début des processus de dégénérescence se traduit par une multiplication des vacuoles, des corps multivésiculaires et des lysosomes. Dans la zone basale, les lobules lipidiques fusionnent. Les vacuoles autophagiques apparaissent environ 30 jours après l'opération. Toutefois le nombre et la taille de ces formations restent plus limités à ce stade que chez les animaux parvenus à une complète maturité sexuelle dans les conditions normales.

\section{2) Evolution des activités enzymatiques (phosphatase acide)}

Détection cytochimique (épitoquie naturelle et expérimentale). - Chez les vers immatures, pouvant être considérés comme témoins, la phosphatase acide est surtout présente dans des vacuoles dont la taille maximum est environ $0,6 \mu \mathrm{m}$ (fig. 2). Une réaction assez discrète est également observée dans les saccules de l'appareil de Golgi. Au cours des premiers stades de l'épitoquie naturelle comme de l'épitoquie expérimentale, l'intensité des réactions de mise en évidence de la phosphatase acide s'accentue. L'enzyme paraît localisée essentiellement dans les saccules de l'appareil de Golgi et dans les vacuoles. Les vacuoles à contenu hydrolytique étant plus nombreuses et de taille plus importante $(0,8$ à $1,2 \mu \mathrm{m})$ que chez les témoins (fig. 13). Ultérieurement les saccules golgiens restent riches en phosphatase acide (fig. 9). L'activité la plus intense est toutefois observée au niveau des citernes de réticulum, souvent en relation avec les lysosomes (fig. 9). Ces lames de réticulum paraissent impliquées dans les processus de séquestration cytoplasmique à l'origine des vacuoles autophagiques. Dans ces organites, la concentration en phosphatase est cependant généralement plus diffuse et d'intensité plus restreinte que dans les citernes de réticulum (fig. 10).

Dosage biochimique. - Une comparaison des stades évolutifs étant difficile à réaliser avec précision au cours de l'épitoquie naturelle, l'évolution biochimique

FIG. 8. - Ver épitoque. Vacuole autophagique (VA) localisée à proximité d'un dictyosome (G.). $\times 23000$.

FIG. 9. - Idem. Mise en évidence de phosphatase acide dans les saccules de l'appareil de Golgi (G), les citernes de réticulum (R) et les lysosomes (Ly) (coupe non colorée). X 30000 .

FIG. 10. - Idem. Phosphatase acide au niveau des saccules golgiens (G). Réaction plus faible au niveau de la vacuole autophagique (VA) (Coupe non colorée). $\times 28000$.

FIG. 11. - Idem. Cellule en dégénérescence (CD) faisant hernie dans la lumière de l'intestin. Noter La disparition complète des microvillosités et des formations apicales denses. Le noyau (N) présente un aspect picnotique. Le cytoplasme renferme de nombreuses vacuoles autophagiques (VA). X 14000 .

FIG. 12. - Epitoquie expérimentale (20 jours d'évolution). Désorganisation des organites apicaux denses (oa). Apparition de nombreuses vacuoles à caractère de lysosomes (V) et de corps multivésiculaires $(\mathrm{cmv}) . \times 29000$.

FIG. 13. - Idem. Mise en évidence de phosphatase acide dans les vacuoles (V). X 15000.

FIG. 14. - Intestin moyen de ver immature. Incorporation de ${ }^{3} \mathrm{H}$-thymidine pendant 24 heures. Marquage de noyaux d'entérocytes (flèches).

FIG. 15. - Intestin moyen de ver en épitoquie expérimentale (20 jours d'évolution). Forte régression du nombre d'entérocytes ayant incorporé de la thymidine pendant 24 heures. Noter la présence de cellules radioactives (cc) dans la cavité coelomique entourant l'intestin. X 140. 
des activités enzymatiques a été réalisée sur des animaux en expérimentation. Les témoins sont représentés par des animaux non décérébrés, sacrifiés à la fin de l'expérience et gardés dans des conditions de jeûne pendant cette durée.

La courbe de la figure 16 montre que les activités hydrolytiques au niveau de l'intestin deviennent de plus en plus importantes en fonction du temps. L'activité phosphatasique acide augmente rapidement au bout de la deuxième semaine après l'ablation du cerveau, et atteint une valeur presque double de celle des témoins. Au bout de quatre semaines, cette activité atteint une très forte valeur représentant environ six fois la valeur des témoins.

Cette évolution des activités hydrolytiques est en corrélation avec les phénomènes de dégénérescence qui se manifestent au niveau de l'épithélium intestinal au moment de l'épitoquie.

\section{3) Evolution des synthèses d'ADN.}

Données autoradiographiques. - Cette étude a été réalisée sur des vers ayant subi une injection de thymidine tritiée dans la cavité coelomique, pour une durée d'incubation de $24 \mathrm{~h}$. Les observations effectuées sur des vers atoques (soumis au jeûne $\left(^{*}\right)$ ) sont comparées à celles des épitoques expérimentaux ayant subi une ablation du cerveau pour une période de 20 jours (fig. 14 et 15).

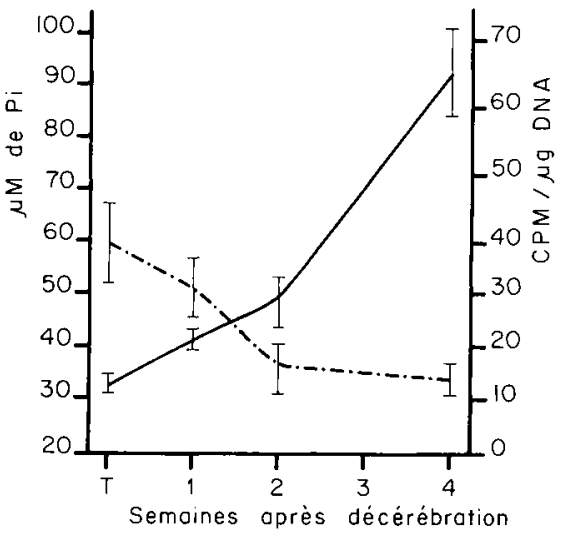

FIG. 16.
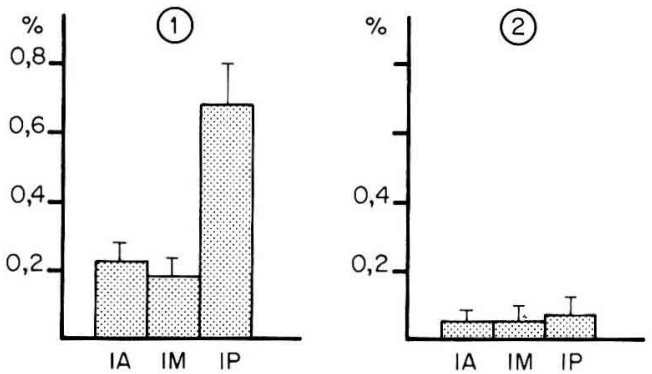

FIG. 17.

FIG. 16. - Evolution en fonction du temps de l'activité phosphatasique acide et des synthèses d'ADN après ablation du cerveau.

- - : Synthèses d'ADN après incorporation de thymidine ${ }^{3} \mathrm{H}$ lexprimées en $\mathrm{cpm} / \mu \mathrm{g}$ de DNA) ; - : Phosphatase acide. L'activité enzymatique est exprimée en $\mu$ mole de phosphate inorganique $(\mathrm{Pi})$ libérée par minute et par $\mathrm{mg}$ de protéines.

$T$ : témoin (chaque mesure correspond au dosage sur 5 vers).

FIG. 17. - Evolution du nombre des mitoses de l'épithelium intestinal après ablation du cerveau. 1 : Vers normaux mais soumis au jeûne pour être dans les mêmes conditions de nutrition que les vers décérébrés ; 2 : Vers décérébrés depuis 20 jours.

Les résultats sont exprimés en \% de noyaux marqués (après incorporation de thymidine $\left.{ }^{3} \mathrm{H}\right)$ par rapport au nombre total de noyaux. IA : intestin antérieur ; $\mathrm{IM}$ : intestin moyen ; IP : intestin postérieur.

(*) Les témoins sont soumis au jeûne car après décérébration les vers ne se nourrissent plus. 
Les résultats sont exprimés en pourcentage de noyaux marqués à la thymidine. Le marquage a été étudié à différents niveaux de l'intestin correspondants aux régions antérieure, moyenne et postérieure. La figure 17 montre que, chez les vers atoques, les synthèses d'ADN varient selon les régions de l'intestin. Le maximum de noyaux marqués est observé dans la région postérieure de l'intestin. Les noyaux marqués sont localisés dans la zone basale de l'épithélium.

En l'absence d'hormone cérébrale, le taux de marquage baisse nettement dans les trois régions de l'intestin.

Données biochimiques. - L'expérimentation a porté sur des lots d'animaux soumis à des conditions identiques à celles du dosage de la phosphatase acide. L'évolution des synthèses d'ADN en condition anhormonale est représentée dans la figure 16. Par rapport aux témoins, les synthèses d'ADN chutent nettement à partir de la première semaine suivant l'ablation du cerveau. Cette régression est très sensible lors de la deuxième semaine. Le taux de synthèse reste ensuite très faible jusqu'à la fin de l'expérience.

\section{Discussion.}

Au cours de l'épitoquie, naturelle ou expérimentale, l'épithélium intestinal des Néréidiens subit une phase d'involution importante. Les microvillosités de l'épithélium disparaissent. Les mitochondries et le réticulum présentent un aspect dilaté. Les synthèses de l'appareil de Golgi s'orientent vers la production de lysosomes primaires. Des portions de cytoplasme sont séquestrées par des membranes de l'appareil de Golgi et du réticulum endoplasmique et forment des vacuoles autophagiques. Ces structures sont alors progressivement dégradées par enrichissement en hydrolases acides résultant de l'incorporation de lysosomes primaires ou secondaires. Cette évolution cytologique est proche de celle décrite au cours de divers processus de dégénérescence autophagique survenant soit dans des muscles d'Insectes (voir revue dans Beaulaton et Lockshin, 1982) soit dans l'intestin des tétards d'Anoures (revue dans Hourdry et Dauca, 1977).

Des processus de rejet de cellules en dégénérescence ont été assez fréquemment signalés, notamment dans l'intestin des Insectes (Andriès, 1977, 1983; Humbert, 1979) et dans des glandes sécrétoires d'Amphibiens (Beaumont et Hourdry, 1976). Chez les Néréidiens, malgré l'absence de renouvellement cellulaire, un épithélium intestinal reste en place au moment de la reproduction (qui sera suivie de la mort de l'animal); mais cet épithélium est aminci et très fragile. Sa rupture représentera chez les femelles une voie d'expulsion des ovocytes.

Lors de l'épitoquie, la chute du taux de renouvellement cellulaire est corrélative des syndromes de dégénérescence de l'épithélium. Chez le ver immature où l'activité cérébrale est élevée, les synthèses d'ADN intestinales sont actives (Punin et Lukyanova, 1984b; notre étude). L'ablation du cerveau détermine une chute des synthèses d'ADN et du nombre des cellules en phase $S$. II semble donc que la (ou les) hormone(s) émise(s) par le cerveau exerce(nt) un rôle stimulateur sur les mitoses intestinales. Cet effet positif se retrouve au niveau des mitoses de la régénération (Durchon et Marcel, 1962). Il s'oppose chez les mâles à celui de 
nature inhibitrice exercé sur les synthèses de DNA préméiotique par l'intermédiaire du contrôle des synthèses de RNA (Bertout, 1983).

Liées à la reproduction, les modifications intestinales de l'épitoquie apparaissent comme un exemple de dégénérescence cellulaire programmée. En effet, les modifications cytologiques observées lors de l'étude expérimentale ne peuvent être une conséquence du jeûne. A l'inverse du cas des Insectes où les altérations provoquées par le jeûne sont considérables (voir revue dans Andriès, 1983), les répercussions sont très discrètes chez les Annélides, ce n'est qu'au bout d'une période excessivement longue 14 à 5 mois) que des formes de dégénérescence apparaissent.

Une relation entre action hormonale et dégénérescence cellulaire a été décrite dans plusieurs modèles biologiques. Les muscles constituent un bon exemple d'organes cibles sensibles à l'action de différentes hormones, à la fois chez les Amphibiens (Fox, 1972) et les Insectes (voir Beaulaton et Lockshin, 1982). Chez les Annélides Polychètes, lors de la stolonisation des Syllidiens (Wissocq, 1970a et b) et de l'épitoquie des Néréidiens (Wissocq, 1977), une partie importante des fibres musculaires subit par dédifférenciation des remaniements structuraux. Mais d'autres fibres présentent des processus de dégénérescence, leur fragmentation sera à l'origine des sarcolytes libérés dans la cavité coelomique et phagocytés par les éléocytes (Dhainaut, 1984).

Au niveau de l'épithélium intestinal, l'induction de la dégénérescence de l'épithélium larvaire sous l'action de l'hormone thyroïdienne a clairement été démontrée chez les Amphibiens (Hourdry et Dauca, 1977). Chez les Insectes, à des degrés variés, l'ecdysone apparaît responsable de la mort cellulaire de l'épithélium larvaire (Radford et Misch, 1971 ; Socha et Sehnal, 1972 ; Andriès, 1979). La Nereis diffère des exemples précédemment cités car la dégénérescence survient lors de la chute d'une hormone stimulatrice et non lors de son apparition. Wyllie et al. (1980) signalent toutefois dans des conditions de chute d'hormone trophique (ACTH), hormones sexuelles) de nombreux cas d'apoptosis, mode de dégénérescence auquel se rattachent selon les auteurs, les processus de dégénérescence liés à la métamorphose. D'autre part, la dégénérescence intestinale chez les Annélides est définitive (sans mise en place d'un nouveau type d'épithélium). La signification physiologique de ce processus n'apparaît d'ailleurs pas clairement. On peut penser qu'à ce moment le ver a accumulé suffisamment de réserves énergétiques. Dans le cadre de la stratégie de la reproduction, tous les remaniements morphogénétiques (muscles, yeux, soies) convergent alors vers une adaptation à la vie pélagique conditionnant la libération des gamètes par essaimage.

Reçu en octobre 1984. Accepté en février 1985.

\section{Références}

ANDRIESS J.-C., 1977. Dégénérescence et rejet cellulaire au niveau du mésentéron d'Aeshna cyanea (Insecte Odonate). Biol. cell., 29, 203-208. 
ANDRIĖS J.-C., 1979. Induction expérimentale de la morphogenèse et de la dégénérescence mésentérique chez Aeshna cyanea (Insecte Odonate). Gen. comp. Endoc., 39, 174-191.

ANDRIĖS J.-C., 1983. L'intestin moyen des Insectes. II. Dynamique cellulaire. Ann. Biol., 22, 261-288.

BEAULATON J., LOCKSHIN R. A., 1982. The relation of programmed cell death to development and reproduction : comparative studies and an attempt at classification. Int. Rev. Cytol., 79. 215-235.

BEAUMONT A., HOURDRY J., 1976. Différents modes de nécrose cellulaire et d'élimination des cellules nécrosées au cours de la métamorphose des Amphibiens Anoures. Bull. Soc. Zool. Fr., 101, 89-94.

BERTOUT M., 1976. Spermatogenèse de Nereis diversicolor Müller (Annélide Polychète). I. Evolution du cytoplasme et élaboration de l'acrosome. J. Microsc. Biol. cell., 25, 88-93.

BERTOUT M., 1983. Endocrine regulation of spermatogenesis in Nereis diversicolor (Annelida, Polychaeta) : experimental study of the control of meiotic differentiation. J. exp. Zool., 226, 151161.

CLARK R.B., 1961. The origin and formation of the heteronereis. Biol. Rev., 36, 199-236.

DHAINAUT A., 1984. Aspects cytophysiologiques des coelomocytes de Néréidiens (Annélides Polychètes). Arch. Anat. microsc., 73, 133-150.

DURCHON M., 1948. Epitoquie expérimentale chez deux Polychètes : Perinereis cultrifera Grübe et Nereis irrorata Malmgrenn. C. R. Acad. Sci, Paris, 227, 157-158.

DURCHON M., 1952. Recherches expérimentales sur deux aspects de la reproduction chez les Annélides Polychètes : l'épitoquie et la stolonisation. Ann. Sci. nat., 11e Sér., 14, 119-206.

DURCHON M., MARCEL R., 1962. Influence du cerveau sur la régénération postérieure chez Nereis diversicolor O.F. Müller (Annélide Polychète). C. R. Soc. Biol., 156, 661-663.

FOX H., 1972. Muscle degeneration in the tail of Rana temporania larvae at metamorphic climax : an electron microscopic study. Arch. Biol., 83, 407-417.

GILES K. W., MYERS A., 1965. An improved diphenylamine method for the estimation of deoxyribonucleic acid. Nature, 206, 93.

HOURDRY J., DAUCA M., 1977. Cytological and cytochemical changes in the intestinal epithelium during anuran metamorphosis. Int. Rev. Cytol., suppl. 5, 337-385.

HUMBERT W., 1979. The midgut of Tomocerus minor Lubbock (Insecta, Collembola) : ultrastructure, cytochemistry, ageing and renerval during a moulting cycle. Cell Tiss. Res., 196, 39-57.

LOCKSHIN R. A., WILLIAMS C. M., 1964. Programmed cell death. II. Endocrine potentiation of the breakdown on the intersegmental muscles of silkmothes. J. Insect Physiol., 10, 643-649.

MILLER F., PALADE G. E., 1964. Lytic activities in renal protein absorption droplets. An electron microscopical cytochemical study. J. Cell. Biol., 23, 519-552.

PORCHET M., 1972. Variation de l'activité endocrine des cerveaux en fonction de l'espèce, du sexe et du cycle vital chez quelques Néréidiens (Annélides Polychètes). Gen. comp. Endoc., 18, 276-283.

PUNIN M. Y., LUKYANOVA E. L., 1984a. Midgut epithelium organization of the errant polychaete Nereis virens. I. Absorptive and secretory cells (en russe). Tsitologiyd, 26, 60-67.

PUNIN M. Y., LUKYANOVA E. L., 1984b. Midgut epithelium organization of the errant polychaete Nereis virens. II. Basal cells (en russe). Tsitologiyá, 26, 188-194.

RADFORD S. V., MISHCH D. W., 1971. The cytological effect of ecdysterone on the midgut cells of the flesh-fly Sarcophaga bullata. J. Cell Biol., 49, 702-711.

SOCHA R., SEHNAL F., 1972. Inhibition of adult development in Tenebrio molitor by Insect hormone and antibiotics. J. Insect Physiol., 18, 317-337.

WATTIAUX R., DE DUVE C., 1956. Tissue fractionation studies. VII. Release of bound hydrolases by means of Triton X-100. Biochem. J., 63, 606-608.

WISSOCO J.-C., 1970a. Evolution de la musculature longitudinale dorsale et ventrale au cours de la stolonisation de Syllis amica Quatrefages (Annélide Polychète). II. La dédifférenciation. J. Microscopie, 9, 1049-1074.

WISSOCQ J.-C., 1970b. Evolution de la musculature longitudinale dorsale et ventrale au cours de la stolonisation de Syllis amica Quatrefages (Annélide Polychète). III. La dégénérescence. J. Microscopie, 9, 1075-1088. 
WISSOCO J.-C., 1977. Evolution de la musculature des Néréidiens (Annélides Polychètes) au cours de l'épitoquie. III. La dédifférenciation des fibres longitudinales. W. Roux's Arch. Develop. Biol., 182, 227-253.

WYLLIE A. H., KERR J. F. R., CURRIE A. R., 1980. Cell death : the significance of apoptosis. Int. Rev. Cytol., 68, 251-306. 